\title{
Physics gets its hands dirty
}

\author{
Is condensed-matter physics becoming more materials-oriented? Or is this just a new wrinkle in an \\ old tradition?
}

Condensed-matter physics is becoming increasingly oriented towards materials science and engineering. That's the conclusion reached by two physicists, Michael Shulman and Marc Warner, after analysing the statistics of abstracts for the main annual (March) meeting of the American Physical Society since 2007 (http://arxiv.org/abs/1502.03103). They enumerated key words used in abstracts to identify trends over the past eight years, and say that during this time the words that are increasing in popularity are often ones associated with specific types of material system, such as 'layer', 'thin', 'organic', 'oxide' and indeed 'material'. In contrast, words or (word fragments) with generally declining popularity include 'superconduct' and 'flux' (as well as, oddly, 'science').

What should we make of this? Probably not too much. As the authors are the first to point out, the analysis is preliminary and its time span limited. It would be good to see it extended over a longer period and expanded to include, say, words in the abstracts of publications in Physical Review Letters, not to mention paying more attention to soft matter rather than primarily solid-state. The present results also paint a slightly confusing picture, taken at face value: condensed-matter physics as a whole has been expanding if one judges from the gradual rise in the total number of abstracts submitted to condensed-matter physics sessions of the March meeting, yet the 'condensed matter' section of the preprint server arXiv has made up a shrinking proportion of the total during that time. There are various possible explanations for the discrepancy.

All the same, if it is qualitatively true that condensed-matter physics has become more materials-focused, it's worth asking why. Are established researchers in the field altering the direction of their work away from abstract theoretical questions such as the origin of high-temperature superconductivity, to take one obvious former preoccupation of theorists - and towards applications of particular materials systems? Although this shift could instead reflect the different interests of young researchers entering the field, old hands

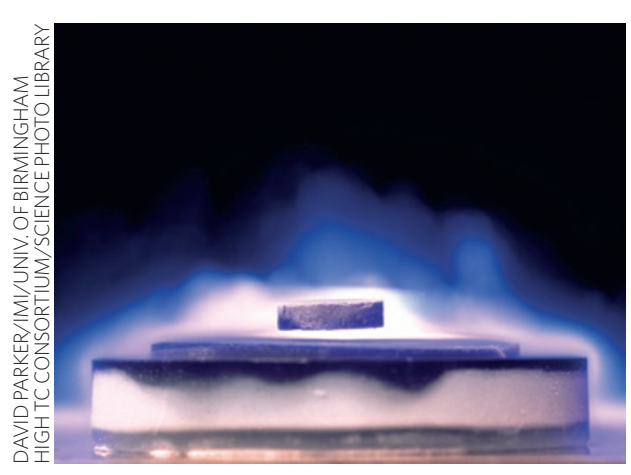

High-temperature superconductivity with cuprate materials has passed its peak. What happens to the physics when the materials move on?

can enjoy fresh challenges too, and can use their knowledge to have insights that the youngsters may miss.

It is tempting to infer that researchers are just following the money: in this increasingly goal-oriented scientific climate, there may be better funding prospects for a project that can promise concrete applications at the end of the line. But might not the trend instead reflect the internal dynamics of the research community, so that funding follows areas deemed 'hot' for other reasons? It's almost sure to be a bit of both, as the example of graphene shows: there are high hopes for applications in electronics and composites, but much of the interest has come from the fundamental physics that this onedimensional system seems to offer. More data on the dynamics and trends of funding priorities might help to separate cause and effect.

In any event, practical applications of materials have always been in the best tradition of condensed-matter physics. Given the enormous contributions that the field has made to society - underpinning the technologies of smart phones and solar energy, say - it's only natural that researchers should have an eye on ensuring that this tradition continues.

Shulman and Warner also found that, in comparison to subjects such as atomic, molecular and optical physics, condensedmatter physics changes fast: the statistics of key words are more volatile. This may be a feature of the field, if only because physicists are intrinsically enthusiastic and interested in new topics, and so respond quickly to materials innovations. This may be the case for pnictide superconductivity, which enjoyed its greatest popularity just before the period of this analysis. Superconductivity is seeing another little surge of interest now owing to topological superconductors.

All fields may have a natural life cycle, but Shulman and Warner wonder if this cycle is shorter in condensed-matter physics than elsewhere, perhaps because it can be stimulated by the discovery of a new material system (carbon nanotubes, say, or magnetic multilayers). Or perhaps it can be particularly hard to get at the high-lying fruits for many of these systems, owing to the complexities of the many-body interactions they present - that, at least, seems to be what has kept a general theory of high-temperature superconductivity out of reach. And high-temperature superconductivity also showed that there is a very low entry barrier for studying exciting new materials if they are relatively easy to synthesize: any lab well equipped with instrumentation can quickly and easily switch direction and still hope to make a useful contribution.

Might there also be a life cycle for condensed-matter physics as a whole? The American Physical Society's condensedmatter division was created only in 1978, from what was formerly the Division of Solid State Physics. Yet Shulman and Warner wonder if it still presents the kind of exciting challenges of 20-30 years ago. No one would claim, however, that the most demanding questions are all answered: perhaps some of them will need to await new techniques or new theoretical methods better able to accommodate the true complexity of the materials. Like chemistry, to some extent condensedmatter physics creates its own subject: our inventiveness (or serendipity) with new materials systems prompts new questions. And to explore the complex physics of these questions, there's really no good alternative to performing experiments on real materials. 